\title{
Preface (Vol 37. Issue 1)
}

\author{
Nadia Magnenat-Thalmann ${ }^{1}$
}

Accepted: 16 October 2020 / Published online: 21 November 2020

(c) Springer-Verlag GmbH Germany, part of Springer Nature 2021, corrected publication 2020

In this issue, we have five papers for a special section selected from the conference VS-Games 2018, three CGI'2020 remaining papers, and six regular papers.

\section{From VS-Games 2028 conferences, 5 best papers have been selected for this special section:}

The first paper is by Carlo Harvey et al and titled: "A comparison between expert and beginner learning for motor skill development in a virtual reality serious game"

The second paper is by Afonso Gonçalves et al and titled: "Effects of prolonged multidimensional fitness training with exergames on the physical exertion levels of older adults" The third paper is by Thomas Tregel et al and titled: "Looking for Charizard: applying the orienteering problem to locationbased games"

The fourth paper is by Ioannis Rallis et al and titled: "Bidirectional long short-term memory networks and sparse hierarchical modeling for scalable educational learning of dance choreographies"

The fifth paper is by Rory Clifford and titled: "Aerial firefighter radio communication perfor-mance in a virtual training system: radio communication disruptions simulated in VR for Air Attack Supervision"

\section{From CGI'2020 Conference, 3 remaining reviewed papers are listed below:}

The first CGI paper is "Total Generalized Variation Based Retinex Image Decomposition" by Chunxue Wang from Dunhuang Academy, Gansu, Huayan Zhang from Tiangong University, Tianjin, and Ligang Liu from University of Science and Technology of China, Anhui, China.

The second CGI paper is "Fine-Grained Talking Face Generation with Video Reinterpretation" by Xin Huang and Mingjie Wang from Memorial University of Newfoundland, and Minglun Gong from University of Guelph, Canada.

Nadia Magnenat-Thalmann

thalmann@miralab.ch

1 MIRALab-CUI, University of Geneva, Battelle, Building A, 7, Route de Drize, Carouge, 1227 Geneva, Switzerland
The third CGI paper is "Modeling Distracted Agents In Crowd Simulations" by Melissa Kremer, from York University, Toronto and Brandon Haworth from University of Victoria, Canada Victoria Mubbasir Kapadia from Rutgers University

\section{The following 6 papers are regular papers:}

The first regular paper is from Mehran Safayani et al and titled:"Multiple answers to a question: a new approach for visual question answering"

The second regular paper is from Wei Cui et al and titled "A single-shot multi-level feature re-used neural network for object detection"

The third paper is from Sunil Kumar et al titled: "Multilevel uncorrelated discriminative shared Gaussian process for multi-view facial expression recognition"

The fourth paper is from Qiang Lu et al titled: "Evaluation on visualization methods of dynamic collaborative relationships for project management"

The fifth paper is from Naoki Hashimoto titled: "Radiometric compensation for non-rigid sur-faces by continuously estimating inter-pixel correspondence"

The sixth paper is from Ali Mansouri et al titled: "Image encryption using shuffled Ar-nold map and multiple values manipulations"

Nadia Magnenat-Thalmann.

Editor-in-Chief.

The Visual Computer.

Publisher's Note Springer Nature remains neutral with regard to jurisdictional claims in published maps and institutional affiliations. 